\title{
An Improved Implementation of Brain Tumor Detection Using Segmentation Based on Hierarchical Self Organizing Map
}

\author{
T.Logeswari and M.Karnan
}

\begin{abstract}
Image segmentation denotes a process of partitioning an image into distinct regions. A large variety of different segmentation approaches for images have been developed. Among them, the clustering methods have been extensively investigated and used. In this paper, a clustering based approach using a Self Organizing Map (SOM) algorithm is proposed for medical image segmentation. This paper describe segmentation method consists of two phases. In the first phase, the MRI brain image is acquired from patient database. In that film artifact and noise are removed. In the second phase (MR) image segmentation is to accurately identify the principal tissue structures in these image volumes. A new unsupervised MR image segmentation method based on fuzzy C-Means clustering algorithm for the Segmentation is presented
\end{abstract}

Index Terms-Image analysis, Segmentation, HSOM, Fuzzy C-Means, Tumor detection

\section{INTRODUCTION}

The brain is a soft, delicate, non-replaceable and spongy mass of tissue. It is a stable place for patterns to enter and stabilize among each other. A tumor is a mass of tissue that grows out of control of the normal forces that regulates growth [21]. Brain tumor is a group of abnormal cells that grows inside of the brain or around the brain. Tumors can directly destroy all healthy brain cells. It can also indirectly damage healthy cells by crowding other parts of the brain and causing inflammation, brain swelling and pressure within the skull. Over the last 20 years, the overall incidence of cancer, including brain cancer, has increased by more than $10 \%$, as reported in the National Cancer Institute statistics (NCIS) The National Brain Tumor Foundation (NBTF) for research in United States estimates that 29,000 people in the U.S are diagnosed with primary brain tumors each year, and nearly 13,000 people die. In children, brain tumors are the cause of one quarter of all cancer deaths. The overall annual incidence of primary brain tumors in the U.S is 11 to 12 per 100,000 people for primary malignant brain tumors, that rate is 6 to 7 per $1,00,000$. In the UK, over 4,200 people are diagnosed with a brain tumor every year (2007 estimates). There are about 200 other types of tumors diagnosed in UK each year. About 16 out of every 1,000 cancers diagnosed in the

T.Logeswari is a Research Scholar, with the Dept of Computer Science, Mother Theresa women's University, Kodaikkanal, India (email: saralogu4uin@gmail.com ).

M.Karnan is with the Department of Computer Science and Engineering, Tamilnadu College of Engineering, Coimbatore, India (email: drmkarnan@gmail.com).
UK are in the brain (or 1.6\%). In India, totally 80,271 people are affected by various types of tumor (2007 estimates).

\section{RELATED WORKS}

The Segmentation of an image entails the division or separation of the image into regions of similar attribute. The ultimate aim in a large number of image processing applications is to extract important features from the image data, from which a description, interpretation, or understanding of the scene can be provided by the machine. The segmentation of brain tumor from magnetic resonance images is an important but time-consuming task performed by medical experts The digital image processing community has developed several segmentation methods[8], many of them ad hoc. Four of the most common methods are: 1) amplitude thresholding, 2) texture segmentation 3) template matching, and 4) region-growing segmentation. It is very important for detecting tumors, edema and necrotic tissues. These types of algorithms are used dividing the brain images into three categories (a) Pixel Based (b) Region or Texture Based (c) Structural Based. Several authors suggested various algorithms for segmentation [3:6:12:13:18:23:25].

Siyal et al described a new method on Fuzzy C-means for segmentation purpose [30] . Phillips,W.E et al described Application of fuzzy C-Means Segmentation Technique for tissue Differentlation in MR Images of a hemorrhagic Glioblastoma Multiforme[24]. S. Murugavalli1 et al , A high speed parallel fuzzy c-mean algorithm for brain tumor segmentation [34]. S. Murugavalli1, An Improved Implementation of Brain Tumor Detection Using Segmentation Based on Neuro Fuzzy Technique [35],. Vaidyanathan $M$ et al described Comparison of Supervised MRI Segmentation methods for Tumor Volume Determination During Therapy[36]. Jayaram K et al described Fuzzy Connectedness and Image Segmentation[15].Kannan et aln describe Segmentation of MRI Using New Unsupervised Fuzzy C mean Algorithm[16] Ruspini, E Described Numerical methods for fuzzy clustering[28]. Dunn, J.C., described A fuzzy relative of the ISODATA process and its use in detecting compact, well Separated clusters[8] . Bezdek, J.C., described Cluster validity with fuzzy sets[5].

\section{IMAGE AQUSTITATION}

Images of a patient obtained by MRI scan is displayed as an array of pixels (a two dimensional unit based on the matrix 
size and the field of view) and stored in Mat lab 7.0.Here, grayscale or intensity images are displayed of default size $256 \times 256$. A grayscale image can be specified by giving a large matrix whose entries are numbers between 0 and 255 , with 0 corresponding, say, to black, and 255 to white. A black and white image can also be specified by giving a large matrix with integer entries. The lowest entry corresponds to black, the highest to white. All MR images were acquired on a $0.5 \mathrm{~T}$ open interventional MRI system (Signa sp). MR Images were transformed on to a Linux network through LAN (KMCH Hospital, India). (All images had $1 \mathrm{~mm}$ slice thickness with $1 \times 1 \mathrm{~mm}$ in plane resolution.

\section{PREPROCESSING}

Noise presented in the image can reduce the capacity of region growing filter to grow large regions or may result as a fault edges. When faced with noisy images, it is usually convenient to preprocess the image by using weighted median filter. Weighted Median (WM) filters have the robustness and edge preserving capability of the classical median filter. WM filters belong to the broad class of nonlinear filters called stack filters. This enables the use of the tools developed for the latter class in characterizing and analyzing the behavior and properties of WM filters[23], e.g. noise attenuation capability. The fact that WM filters are threshold functions allows the use of neural network training methods to obtain adaptive WM filters

\section{Hsom With FCM Image SEgEMENTAtion}

Segmentation is an important process to extract information from complex medical images. Segmentation has wide application in medical field[1;2;11;24].The main objective of the image segmentation is to partition an image into mutually exclusive and exhausted regions such that each region of interest is spatially contiguous and the pixels within the region are homogeneous with respect to a predefined criterion. Widely used homogeneity criteria include values of intensity, texture, color, range, surface normal and surface curvatures. During the past many researchers in the field of medical imaging and soft computing have made significant survey in the field of image segmentation [4;9;17;29]. Image segmentation techniques can be classified as based on edge detection, region or surface growing, threshold level, classifier such as Hierarchical Self Organizing Map (HSOM), and feature vector clustering or vector quantization[33]. Vector quantization has proved to be a very effective model for image segmentation process [32]. Vector quantization is a process of portioning $n$-dimensional vector space into $M$ regions so as to optimize a criterion function when all the points in each region are approximated by the representation vector $\mathrm{Xi}$ associated with that region. There are two processes involved in the vector quantization: one is the training process which determines the set of codebook vector according to the probability of the input data, the other is the encoding process which assigns input vectors to the code book vectors. Vector quantization process has been implemented in terms of the competitive learning neural network (CLNN)[7]. Self Organizing Map (SOM)[10] is a member of the CLNNs and this can be the best choice when implementing vector quantization using neural network[22]. The importance of SOM for vector quantization is primarily due to the similarity between the competitive learning process employed in the SOM and the vector quantization procedure.

HSOM combine the idea of regarding the image segmentation process as one of data abstraction where the segmented image is the final domain independent abstraction of the input image. The hierarchical segmentation process for a hierarchical structure is called abstraction tree. The abstraction tree bears some resemblance to the major familiar quad tree data structure [15] used in the several image processing and image analysis algorithms. The researchers in this field have used SOM or HSOM separately as one of the tool for the image segmentation of MRI brain for the tumor analysis. In this paper, we propose a hybrid technique combining the advantages of HSOM was implemented for the MRI image segmentation Clustering is the process of grouping a data set in a way that the similarity between data within a cluster is maximized while the similarity between data of different clusters is maximized[19,31] and is used for pattern recognition in image processing.

The below flowchart represent method of MRI brain image segmentation using Hierarchical self organizing map(Hsom) with FCM(Fuzzy C Means). The below figure shows the flow of work in Hsom with FCM. In image acquisition process $\mathrm{MR}$ brain image is loaded into MATLAB 7.0.in the form of matrix . Next Initialize the variables sigma, weight vector and winning neuron .In that Calculate the neighborhood function, weight vector and winning neuron .Here neuron is the input and winning neuron is the output of HSOM After that Calculate the Dissimilarity function of Maximum and Minimum, Euclidean Distance function, Cluster Points, Cluster center of Som .Here winning neuron is the input (Acquired by Som) and adaptive threshold is the output(Obtained by FCM) if the (Current neuron>= adaptive threshold) then it is suspicious region Other wise neglect it.

\section{IMPLEMENTATION OF HSOM AND FCM ALGORTHIM}

A self-organizing map consists of components called nodes or neurons. Associated with each node is a weight vector of the same dimension as the input data vectors and a position in the map space. The usual arrangement of nodes is a regular spacing in a hexagonal or rectangular grid. The self-organizing map describes a mapping from a higher dimensional input space to a lower dimensional map space. The procedure for placing a vector from data space onto the map is to find the node with the closest weight vector to the vector taken from data space and to assign the map coordinates of this node to our vector. Euclidean to all weight vectors is computed. The neuron with weight vector most similar to the input is called the best matching unit (BMU). The weights of the BMU and neurons close to it in the SOM lattice are adjusted towards the input vector. The magnitude of the change decreases with time and with distance from the BMU. Among the wide range of possible tools, the 
self-organizing map (SOM) is one of the most effective [20].

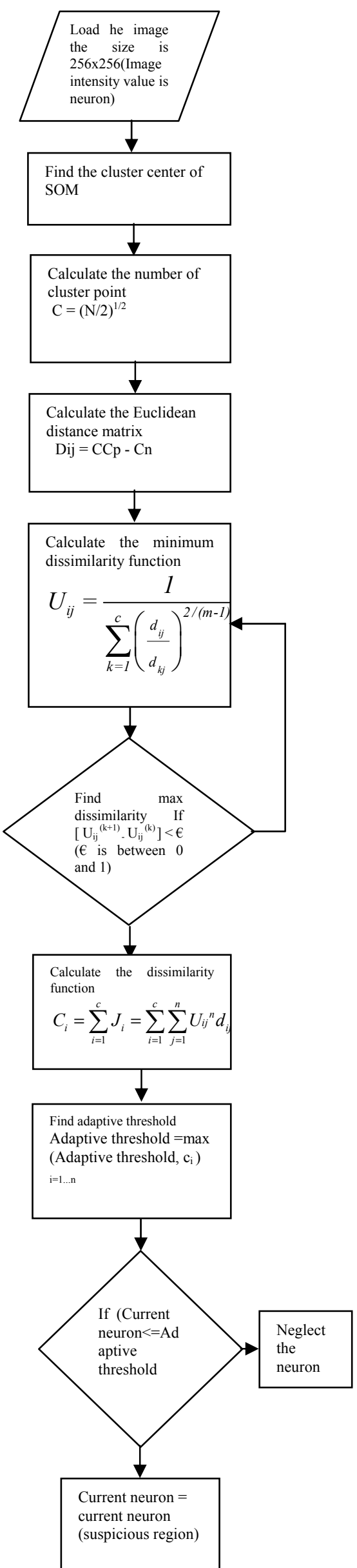

Fig(1) Flowdigram for detection of Brain Tumor
The Self-Organizing Map as a special clustering tool provides a compact representation of the data distribution, has been widely applied in the visualization of high-dimensional data. In $[2,15]$ fuzzy clustering combined with SOM is used to project the data to lower dimensions. The most interesting approach has been presented in [14, $26,27]$ where a fuzzy self-organizing map is developed based on the modifications of the fuzzy c-means functional. The update formula for a neuron with weight vector $\mathrm{Wv}(\mathrm{i})$ is

$$
\begin{gathered}
\mathrm{Wv}(\mathrm{i}+1)=\mathrm{Wi}(\mathrm{i}+1)=\mathrm{wi}(\mathrm{i})+\operatorname{hci}(\mathrm{I}) *[(\mathrm{x}(\mathrm{i})- \\
\mathrm{w}(\mathrm{i}))]
\end{gathered}
$$

Here hci is neighborhood function to calculate it

$$
\mathrm{h}(\mathrm{i})=\mathrm{h}(\mathrm{rc}-\mathrm{r} 1) * \mathrm{a}(\mathrm{i}) * \text { alpha }
$$

Here rc-r1=current neuron-next current neuron

Initialize the variable

$$
a(i)=\operatorname{sigma} 0 * \exp (-\mathrm{i} / \mathrm{nsm})
$$

Sigma $=$ number of neighborhood pixels ( 8 or 24 or 48 or $80 \quad$ or $\quad \begin{aligned} & 120) \text { if } \quad \text { sliding windoe } \\ & =8),(5 * 5=24),(7 * 7=48),(9 * 9=80),(11 * 11=120)\end{aligned}$
Sigma $N=$ Sigma $0 * \exp (-\mathrm{i} /$ taul $)$

Taul= total number of pixels $/ \log ($ neighborhood number of pixel)

Similarly find the sigma value for each and every pixel This process is repeated for each input vector for a (usually large) number of cycles $\lambda$. The network winds up associating output nodes with groups or patterns in the input data set. If these patterns can be named, the names can be attached to the associated nodes in the trained net. The update formula for a neuron with winning neuron is

Step 1:The winning neuron formula is

$$
\|\mathrm{x}-\mathrm{wc}\|=\operatorname{maxi}\{[=\mathrm{x}-\mathrm{wi}]\}
$$

$\mathrm{X}$ is a neuron,wi is the weight vector

This is the winning neuron equation of HSOM giving input to the FCM Based on the previous equation, the first step of the regularized FCM-HSOM algorithm is the following:

Step 2: Calculate the cluster centers.

$$
\mathrm{C}=(\mathrm{N} / 2) 1 / 2
$$

Step 3: Compute the Euclidean distances

$$
\mathrm{Dij}=\mathrm{CCp}-\mathrm{Cn}
$$

Step 4: Update the partition matrix

$$
U_{i j}=\frac{1}{\sum_{k=l}^{c}\left(\frac{d_{i j}}{d_{k j}}\right)^{2 /(m-l)}}
$$

(Repeat the step 4)

Until Max[ $|\operatorname{Uij}(\mathrm{k}+1)-U i j k|]<€$ is satisfied

Step 5: Calculate the average clustering points.

$$
C_{i}=\sum_{i=1}^{c} J_{i}=\sum_{i=1}^{c} \sum_{j=1}^{n} U_{i j}^{n} d_{i j}^{2}
$$

Step 6: Compute the adaptive threshold

Adaptive threshold $=\max$ (Adaptive threshold, ci ) $\mathrm{i}=1$...n In the first step, the algorithm select the initial cluster from Hsom Clustering algorithm. Then, in later step after several iteration of the algorithm, the final result converges to actual cluster center achieved and it is very important for an FCM algorithm.

\section{RESUlT AND ANALYSIS}

\begin{tabular}{|l|l|l|l|l|l|}
\hline Value / & $3 \times 3$ & $5 \times 5$ & $7 \times 7$ & $9 \times 9$ & $11 \times 11$ \\
\hline
\end{tabular}




\begin{tabular}{|l|l|l|l|l|l|}
\hline $\begin{array}{l}\text { Neighborhood } \\
\text { pixels }\end{array}$ & & & & & \\
\hline $\begin{array}{l}\text { Adaptive } \\
\text { threshold }\end{array}$ & 184.07 & 152.09 & 149.70 & 141.15 & 133.22 \\
\hline $\begin{array}{l}\text { Number of } \\
\text { segmented } \\
\text { cells }\end{array}$ & 883 & 3732 & 4319 & 7417 & 12025 \\
\hline $\begin{array}{l}\text { Execution } \\
\text { time }\end{array}$ & 31.83 & 33.14 & 29.50 & 27.02 & 28.364 \\
\hline Weight & 28.28 & 51.22 & 55.05 & 74.56 & 93.91 \\
\hline
\end{tabular}

Table1 shows the result of image segmentation of Hsom .In any computer aided analysis, the execution time is one of the important parameters of medical image segmentation .In these result, we have calculated the number of tumor cells of different neighborhood pixel of $3 \times 3,5 \times 5$, $7 \times 7,9 \times 9,11 \times 11$ windows. In that $3 \times 3$ window is chosen based on the high contrast than $5 \times 5,7 \times 7,9 \times 9$, and $11 \times 11$.

Table 1:, Adaptive threshold No of segmented pixel, Execution time, weight

Figure 3 shows the tested segmented image with various neighborhood
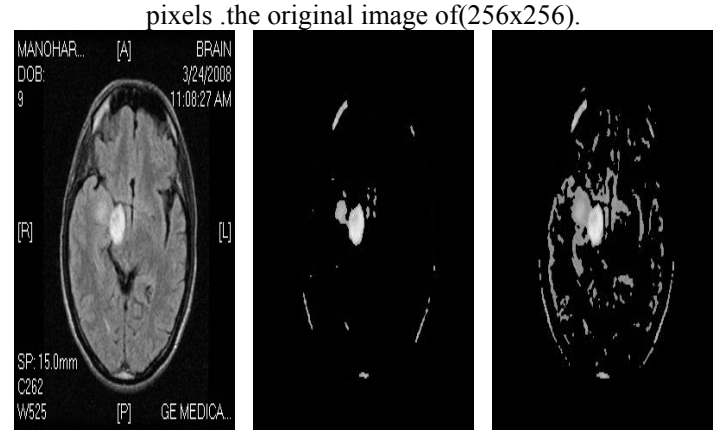

$$
\text { Input }
$$

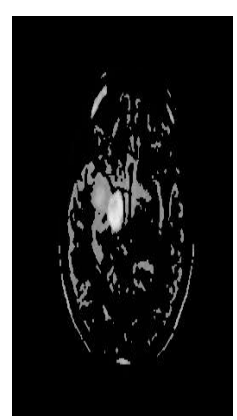

Output

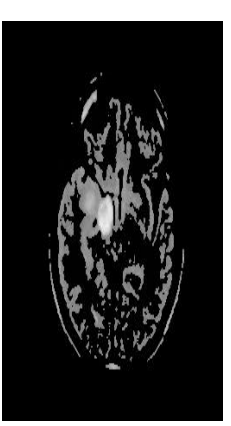

Output $(5 \times 5)$

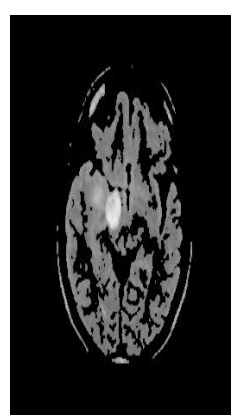

Output

Output

(9x9)

Output

$(11 \times 11)$

Figure (3) A input image (256x256), Outpu3x3, Output 5x5 Output 7x7 Output 9x9 Output 11x11

\section{PERFORMANCE ANALYSIS}

It is very difficult to measure the performance of enhancement objectively. If the enhanced image can make observer perceive the region of interest better, then we can say that the original image has been improved

Here we are giving input image in that neighborhood pixel of $3 \times 3,5 \times 5,7 \times 7,9 \times 9,11 \times 11$ windows are analyzed. In that $3 \times 3$ window is chose based on the high contrast than $5 \times 5$, $7 \times 7,9 \times 9$, and $11 \times 11$. Figure (4c) shows the weight vector for HSOM is $3 \times 3$ is $14,5 \times 5$ is $8,7 \times 7$ is $15,9 \times 9$ is 23 and $11 \times 11$ is 32 .Figure (4a) shows the Execution time in HSOM of $3 \times 3$ is $13.76,5 \times 5$ is $14.96,7 \times 7$ is $15.20,9 \times 9$ is 11.05 and

$11 \times 11$ is 11.53 . Figure (4b) shows the number of segmented pixel in HSOM of $3 \times 3$ is $795,5 \times 5$ is $1073,7 \times 7$ is $1285,9 \times 9$ is 1594 and $11 \times 11$ is 1881 . (4c) shows the wining neuron for HSOM is $3 \times 3$ is $209,5 \times 5$ is $201,7 \times 7$ is $194,9 \times 9$ is 186 and $11 \times 11$ is 177 .

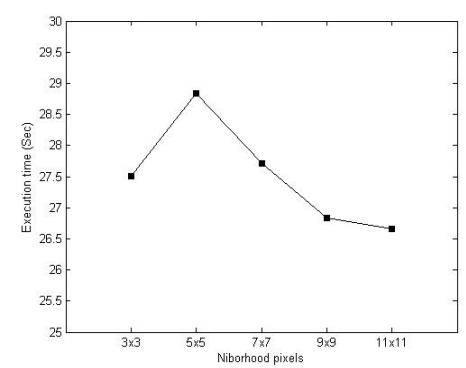

HSom with FCM type of segmentation

Fig(4a) relationship between execution time and neighborhood pixels

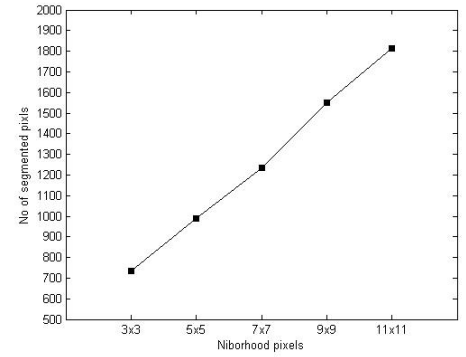

HSom with FCM type of segmentation

Fig(4b) relationship between no of segmented pixel and neighborhood pixels

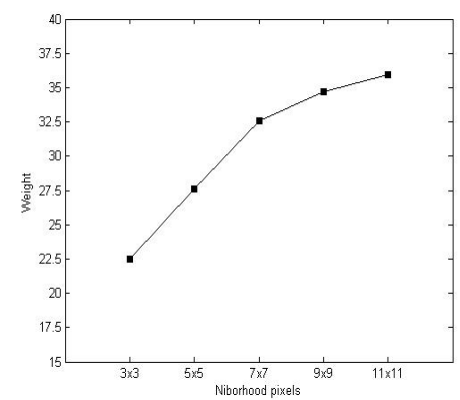

HSom with FCM type of segmentation Fig(4c) relationship between weight and neighborhood pixels

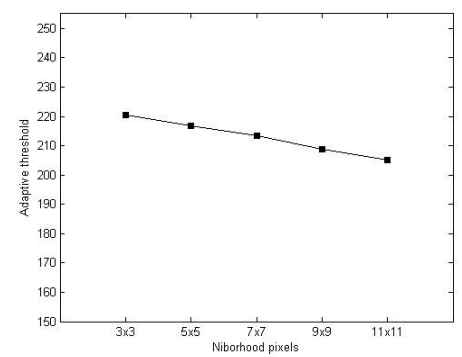

HSOM with FCM type of segmentation

Fig(4d) relationship winning neuron and neighborhood pixels

In above $3 \times 3,5 \times 5,7 \times 7,9 \times 9,11 \times 11$ windows are analyzed in that $3 \times 3$ window is chosen based on the high contrast than $5 \times 5,7 \times 7,9 \times 9$, and $11 \times 11$.

\section{CONCLUSION}

A fuzzy based segmentation process to detect brain tumor was implemented. In that performance of the MRI image in terms of weight vector, execution time and tumor pixels detected We have described several methods in medical image processing and discussed requirements and properties of techniques in brain tumor detection. This paper are used to give more information about brain tumor detection and 
segmentation of HSOM with FCM is given. In this paper various step in detection of automatic system like (a) Image capturing (b) Image segmentation (c) Performance Evaluation are discussed.

\section{ACKNOWLEDGEMENT}

The author wishes to thank Doctor Pankaj Metha for his helpful suggestion on tumor recognition with his knowledge and experience in medicine area. The author also thanks the hospital of $\mathrm{KMCH}$ in Coimbatore ,India .

\section{REFERENCE}

[1] [Aaron Lefohn, Joshua Cates, Ross Whitaker,: "Interactive GPU-Based level sets for 3D Brain Tumor Segmentation",April 16,2003.

[2] Ahalt, S.C., A.K. Krishnamurthy, P. Chen and D.E.Melton, 1990. "Competitive learning algorithms for Vector quantization", Neural Networks 3 (3): 277-290.

[3] Aidyanathan M, Clarke L.P, Velthuizen R.P, Phuphanich S, Bensaid A.M, Hall L.O, Bezdek J.C, Greenberg H, Trotti A, Silbiger M,:"Comparison of Supervised MRI Segmentation methods for Tumor Volume Determination During Therapy",Pergamon,Magnetic Imaging,vol.13,no.5,pp,719-728,1995.

[4] Alirezaie, J., M.E. Jernigan and C. Nahmias, 1997."Neural Network based segmentation of MagneticResonance Images of the Brain", IEEE Trans. Nuclear Science, 44 (2): 194-198.

[5] Bezdek, J.C., 1974. "Cluster validity with fuzzysets", Cybernetics, 3: 58-73.

[6] Chunyan Jiang,Xinhua Zhang,Wanjun Huang,Christoph Meinel.."Segmentation and Quantification of Brain Tumor,"IEEE International conference on Virtual Environment,Human-Computer interfaces and Measurement Systems, USA, 12-14, July 200.

[7] DeSieno, D., 1988. "Adding a conscience to competitive learning", Proceeding of IEEE the Second International Conference on Neural networks(ICNN88) 1: 117- 124.

[8] Dunn, J.C., 1973.” A fuzzy relative of the ISODATA process and its use in detecting compact, well Separated clusters",Journal of Cybernetics, 3: 32-15.

[9] Fu, K.S. and J.K. Mui, 1981." A survey on image segmentation", Pattern ecognition, 13: 3-16.

[10] Gonzalez, R.C. and R.E. Woods, "Digital image processing, Pearson Education", 2002.

[11] Haralick, R.M. and L.G. Shapiro, 1985. "Survey, image segmentation techniques", Computer Vision, Graphics Image Process, 29: 100-132[9]

[12] hanShen,WilliamSandham,MalcolmGranet,Annette Sterr.:"MRI Fuzzy Segmentation of Brain Tissue Using Neighbourhood Attraction with Neural-Network Optimization",IEEE Transcations on Information Technology in BiomedicineVol.9,No.3,Sep 2005.[22]

[13] Hillips,W.E, Velthuizen R.P, Phuphanich S, L.O, Clarke L.P, Silbiger,:"Application of fuzzy C-Means Segmentation Technique for tissue Differentlation in MR Images of a hemorrhagic Glioblastoma Multiforme “, Pergamon,Megnetic Resonance Imaging, Vol.13, 1995[10].

[14] Hung, M.C. and D.L. yang, 2001. "An Efficient Fuzzy C means Clustering Algorithm”, Data mining, ICDM 2001, Proceedings IEEE international conference on pp: 225-232.[22]

[15] Jayaram K.Udupa,Punam K.Saha,."'Fuzzy Connectedness and Image Segmentation", Proceedings of the IEEE, vol.91,No 10, Oct 2003.

[16] Kannan S.R,:"Segmentation of MRI Using New Unsupervised Fuzzy C mean Algorithm",ICGST,2005.

[17] Kohonen, T., 1988. "Self-Organization and Associative Memory", 2nd Edition (Springer-Verlag, Berlin, Germany.

[18] [Kohonen..T "The self-organizing map". Proceedings of the IEEE, $(78(9)): 1464-1480,1990 .[6]$

[19] Kwok, T., R. Smith, S. Lozano and D. Taniar,2002. "Parallel fuzzy c-means clustering for largedata sets", In Burkhard Monien and Rainer Feldmann, editors, EUROPAR02, 2400: 365-314.

[20] Mahamed, N.A., M.N. Ahmed and A. Farag, "Modified fuzzy c-mean in medical image Segmentation", in Proceedings of IEEE International Conference on Acoustics, Speech, and Signal Pocessing, Piscataway, NJUSA,6: 3429-3432.[20]
[21] Lin, W., E. Tsao and C. Chen, 1991. "Constraint satisfaction neural networks for image segmentation", In: T.Kohonen, K. Mkisara, 0. Simula and J. Kangas (eds.), Artificial Neural Networks (Elsevier Science Publishers), pp: 1087-1090.[14]

[22] Naylor, J. and K.P. Li, 1988. "Analysis of a neural network algorithm for vector quantization of speech Parameters", Proceeding of the 1st Annual INNS Meeting, pp: 310-315

[23] Pal, N.R. and S.K. Pal, 1993." A review on image segmentation techniques", Pattern Recognition 26(9): 1277-1294.

[24] Parra, C.A., K. Iftekharuddin and R. Kozma, 2003."Automated Brain Tumor segmentation and pattern recognition using ANN", Computational Intelligence Robotics and Autonomous Systems.

[25] Phillips,W.E, Velthuizen R.P, Phuphanich S, L.O, Clarke L.P, Silbiger, ,"Application of fuzzy C-Means Segmentation Technique for tissue Differentlation in MR Images of a hemorrhagic Glioblastoma Multiforme “, Pergamon,Megnetic Resonance Imaging, Vol.13, 1995.

[26] Rahmi, S., M. Zargham, A. Thakre and D. Chhillar,2004. "A Parallel Fuzzy C-Mean Algorithm for Image segmentation Fuzzy information", processing NAFIPS 04, IEEE Annual meeting, 1: 234-237.

[27] R.D Pascal-Marqui, A.D. Pascual Montano, K. Kochi, and J.M. Carazo. "Smoothly distributed fuzzy c a new self organizing map". Pattern Recognition,(34):2395-2402, 2001.

[28] Ruspini, E., 1970. "Numerical methods for fuzzy clustering", Information Sciences, 2: 319-350.

[29] Sahoo, P.K., S. Soltani, A.K.C. Wong and Y.C. Chen, 1988. "A survey of thresholding techniques", Computer Vision, Graphics Image Process. 41: 233-260.

[30] sai .C, Manjunath B.S,Jagadeesan.R,:"Automated Segmentation of brain MR Images",Pergamon,Pattern Recognition, Vol 28, No 12, March 1995.

[31] Siyal M.Y,Lin yu,:"An Intelligent modified fuzzy C-means based algorithm for bias estimation and Segmentation of brain MRI",Elsevier,Pattern Recognition Letters,26,2005.

[32] Siyal M.Y,Lin yu,:"An Intelligent modified fuzzy C-means based algorithm for bias estimation and Segmentation of brain MRI",Elsevier,Pattern Recognition Letters,26,2005.

[33] Scherf, A. and G. Roberts, 1990. "Segmentation using neural networks for automatic thresholding", in: S. Rogers (ed.), Proc. SPIE Conference on Applications of Artificial Neural Networks (Orlando, FL, 1294), pp: 118-124.

[34] S. Murugavalli1 , V. Rajamani," An Improved Implementation of Brain Tumor Detection Using Segmentation Based on Neuro Fuzzy Technique" Journal of Computer Science 3 (11): 841-846, 2007

[35] S. Murugavalli1 , V. Rajamani," "A high speed parallel fuzzy c-mean algorithm for brain tumor segmentation", BIME Journal, Volume (06), Issue (1), Dec., 2006.

[36] [Vaidyanathan M, Clarke L.P, Velthuizen R.P, Phuphanich S, Bensaid A.M, Hall L.O, Bezdek J.C, Greenberg H, Trotti A, Silbiger ,:"Comparison of Supervised MRI Segmentation methods for Tumor Volume Determination During Therapy",Pergamon,Magnetic Resonance Imaging,vol.13,no.5,pp,719-728,1995. 